\title{
Article \\ Generation of Data-Driven Expected Energy Models for Photovoltaic Systems
}

\author{
Michael W. Hopwood ${ }^{1,2}$ and Thushara Gunda ${ }^{1, *(D)}$ \\ 1 Sandia National Laboratories, Albuquerque, NM 87123, USA; mwhopwo@sandia.gov \\ 2 Department of Statistics and Data Science, University of Central Florida, Orlando, FL 32826, USA \\ * Correspondence: tgunda@sandia.gov
}

\section{check for}

updates

Citation: Hopwood, M.W.; Gunda, T. Generation of Data-Driven Expected Energy Models for Photovoltaic Systems. Appl. Sci. 2022, 12, 1872. https://doi.org/10.3390/app12041872 Academic Editor: Giovanni Petrone

Received: 23 December 2021

Accepted: 31 January 2022

Published: 11 February 2022

Publisher's Note: MDPI stays neutral with regard to jurisdictional claims in published maps and institutional affiliations.

Copyright: () 2022 by the authors Licensee MDPI, Basel, Switzerland. This article is an open access article distributed under the terms and conditions of the Creative Commons Attribution (CC BY) license (https:// creativecommons.org/licenses/by/ $4.0 /)$.

\begin{abstract}
Although unique expected energy models can be generated for a given photovoltaic (PV) site, a standardized model is also needed to facilitate performance comparisons across fleets. Current standardized expected energy models for PV work well with sparse data, but they have demonstrated significant over-estimations, which impacts accurate diagnoses of field operations and maintenance issues. This research addresses this issue by using machine learning to develop a data-driven expected energy model that can more accurately generate inferences for energy production of PV systems. Irradiance and system capacity information was used from 172 sites across the United States to train a series of models using Lasso linear regression. The trained models generally perform better than the commonly used expected energy model from international standard (IEC 61724-1), with the two highest performing models ranging in model complexity from a third-order polynomial with 10 parameters $\left(R_{a d j}^{2}=0.994\right)$ to a simpler, second-order polynomial with 4 parameters $\left(R_{a d j}^{2}=0.993\right)$, the latter of which is subject to further evaluation. Subsequently, the trained models provide a more robust basis for identifying potential energy anomalies for operations and maintenance activities as well as informing planning-related financial assessments. We conclude with directions for future research, such as using splines to improve model continuity and better capture systems with low $(\leq 1000 \mathrm{~kW}$ DC) capacity.
\end{abstract}

Keywords: photovoltaic systems; expected energy models; fleet-scale; lasso regression; performance modeling; machine learning

\section{Introduction}

The increasing penetration of photovoltaic (PV) systems within the energy markets has established the need for evaluating and ensuring high system reliability. In particular, a large emphasis has been placed on monitoring algorithms that can contextualize observed energy generation at a site with information about how the system would have performed in a nominal state [1]. The latter are commonly estimated through expected energy models. Expected energy models are incorporated into many PV performance monitoring tasks, including anomaly detection [2-5], financial planning [6], fleet-level (site vs. site) comparisons [7], degradation analysis [7], and the evaluation of extreme weather effects [8]. The comparison of observed energy values to those derived from expected energy models serves as the basis for informing both tactical (i.e., short-term tasks such as field repair) and strategic (i.e., long-term activities such as site planning) operations and maintenance (O\&M) activities.

Expected energy models can vary from asset-level to site-level estimates [9]. Assetlevel models typically focus on using parameters provided by the manufacturer (e.g., maximum power) $[9,10]$. However, such approaches do not always work well for in-field performance since the parameters were developed in standardized test conditions and thus do not reflect operational conditions [11]. In response to these limitations, empirical methods that use field observations and regression methods have emerged to derive parameters across non-standardized test conditions (e.g., [12,13]). At the site-level, most expected 
energy models leverage the correlation between power production and meteorological covariates $[14,15]$. For example, the standard expected energy model from the International Electrotechnical Commission (IEC) uses irradiance and site capacity information to develop an expected energy estimate [15]. Similarly, the PVUSA model trains a regression model for a given site by estimating power production using local irradiance, temperature, and wind speed conditions [16]. Industry research shows that most expected energy estimates tend to be overestimate production by a median of $3 \%$ but could be up to $20 \%$ [17]. Although the mismatch between observed and expected generation are well-recognized [18], limited attention has been given to date for improving the accuracy of expected energy models at the site-level, especially suited for fleet-level (i.e., site vs. site) comparisons.

This work aims to address this knowledge gap by generating a standardized, interpretable data-driven expected energy model that can be used for fleet-level comparisons. Although gradient-boosted and neural network-based methods have demonstrated significant successes for output performance [19-21], they often lack in model interpretability. In particular, models with high complexity can hide prediction biases or other vulnerabilities [22]. Thus for this work, we opted for more interpretable, regression-based models to increase the transparency of the implemented methods. In addition to identifying a more robust alternate for expected energy modeling, the associated publication of code used for training models (in the open source software pv0ps) enables the extension of these methods to develop site-specific expected energy models for PV systems anywhere in the world or to other renewable energy systems. Such advancements in expected energy model estimates are needed to continue supporting better planning and field O\&M activities, both of which ultimately influence the sustainability of PV sites. The following sections describes the data processing and model construction activities (Section 2), the performance of trained models (Section 3), and summarize primary findings (Section 4).

\section{Methodology}

The data-driven expected energy model training activities were supported by Sandia National Laboratories' PV Reliability, Operations, and Maintenance (PVROM) database [23]. Information about the PVROM database, as well as data processing, model training, and model evaluations, are described in the following Sections.

\subsection{Data}

The PVROM database contains 1.3 million data points of hourly production data across 176 sites in the United States [23], spanning multiple states (Figure 1) and generally ranging between 2017 and 2020. The database contains hourly measurements of expected energy in kiloWatt-hours (kWh), irradiance (Watts per square meter; $\frac{\mathrm{W}}{\mathrm{m}^{2}}$ ), ambient temperature, and module temperature; site-level direct current (DC) capacity is provided by the industry partners. The DC capacity $\left(C_{D C}\right)$ for the sites within the database span from 37.8 kilowatts $(\mathrm{kW})$ to $130,000 \mathrm{~kW}$; a majority of the sites (140) are under 10,000 kW, with 67 of those sites under $1000 \mathrm{~kW}$. A subset of the sites (100) contain industry-partner-provided expected energy estimates generated from proprietary models; these values serve as a basis for model validation activities (see Section 2.5). 


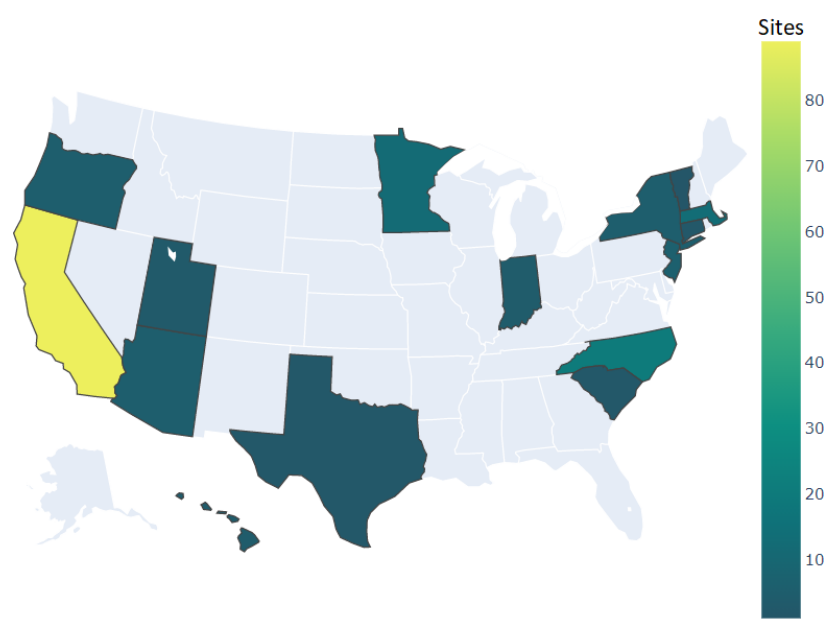

Figure 1. Geographical coverage of sites within the PVROM database. A majority of the sites are located in California.

\subsection{Preprocessing}

Data quality issues stemming from measurement errors and system anomalous conditions (reflecting local field failures, such as communication loss) could introduce signal variations in field data that would hinder model performance. Problematic data convolute the relationships between features, making it more difficult to measure the true parameter estimates; these potential irreducible errors are decreased through numerous data quality filters (Figure 2). Missing values (i.e., NaN or None values) were removed prior to applying data quality filters. An evaluation of these missing values revealed that a majority of them $(\sim 88 \%)$ occurred during nighttime hours ( 7 p.m. to 8 a.m.), indicating that some sites captured night-time entries as null (Figure 2). After removing these missing values, $\sim 900 \mathrm{~K}$ data points remained, which were then subject to a series of data quality filtering steps.

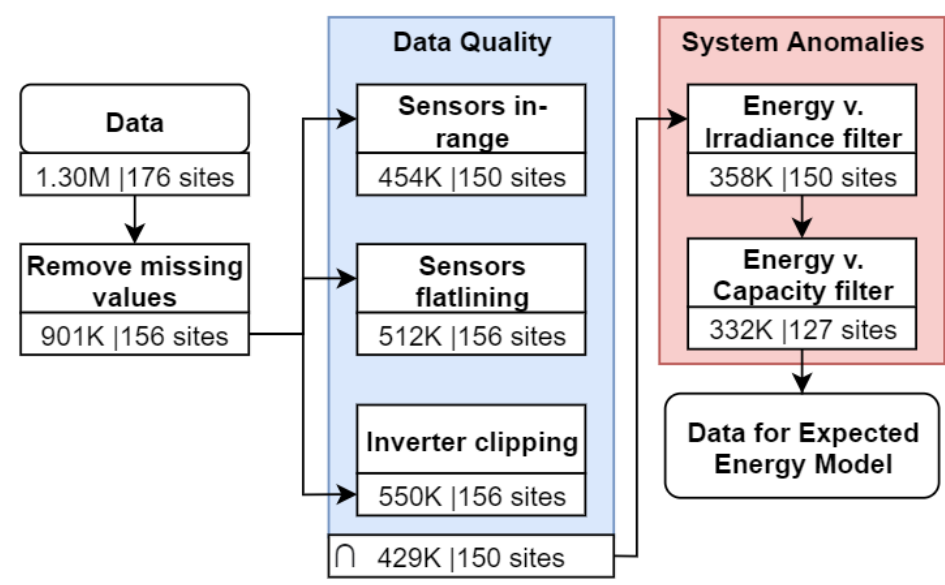

Figure 2. Data preprocessing activities included both data quality- and anomaly-related filters. Data quality filters were conducted independently; only data points that passed all quality-based filters were subject to the anomaly-based filters.

Data were filtered to ensure they are within nominal sensor ranges, using thresholds following [24] and the IEC 61724-1 standard [15]. Specifically, we retained data that met the following criteria:

- $20 \frac{\mathrm{W}}{\mathrm{m}^{2}} \leq$ Irradiance $(I) \leq 1500 \frac{\mathrm{W}}{\mathrm{m}^{2}}$ ；

- $\operatorname{Energy}(E)>0 \mathrm{kWh}$;

- Ambient temperature $\left(T_{a m b}\right) \leq 50{ }^{\circ} \mathrm{C}$ and module temperature $\left(T_{\text {mod }}\right) \leq 90{ }^{\circ} \mathrm{C}$. 
Wind speed was not consistently available from partners and thus was excluded from analysis. Although available temperature data were used in the preprocessing steps, they are not used as a predictor variable in the regression models, since they ate not included in current standard models [15].

Flatlining values-determined by periods where consecutive data changed by less than a threshold-were flagged for removal using the pecos package [25], which follows the IEC 61724-3 standard [26]. Specifically, four consecutive hours with either $\Delta E<0.01 \%$ of the site's capacity or $\Delta I<10 \frac{\mathrm{W}}{\mathrm{m}^{2}}$ were filtered. Lastly, inverter clipping, which occurs when the DC energy surpasses an inverter's DC energy rating, was addressed by mathematically observing plateaus in the energy signal using the pvanalytics package [27]. Dropping energy measurements during inverter clipping, which manifest as a static value across high irradiance levels, would create a better linear fit. After data quality checks, $429 \mathrm{~K}$ data points across 150 sites remained (Figure 2).

Data points that passed all quality checks were also assessed for system-level anomalies. These anomalies likely reflect abnormal operating conditions (i.e., local failures) and thus require removal to ensure the trained baseline energy models reflect nominal system performance. Anomalous entries were detected using a comparison of observed energy to irradiance and site capacities (Figure 3). The comparison of observed energy and irradiance filter focuses on removing data where the E-I ratio $(\lambda)$ is outside its nominal distribution by 3 standard deviations $\left\{\lambda: \lambda<\mu_{\lambda}-3 \sigma_{\lambda} \cup \lambda>\mu_{\lambda}+3 \sigma_{\lambda}\right\}$, where $\mu_{\lambda}$ and $\sigma_{\lambda}$ are the mean and standard deviation of the E-I ratio, respectively [28]. This filter was implemented for each site separately to capture site-specific variations (including system capacity) and resulted in the removal of $70 \mathrm{~K}$ data points (Figure 2). The second system anomaly filter focused on removing sites with mismatches between observed energy and site capacity. Namely, if a site's maximum recorded energy was over $1.2 \times C_{D C}$ or under $0.7 \times C_{D C}$, then all data points for that site were excluded from subsequent analysis. This method filtered 23 sites; $50 \%+$ of these sites were under $1000 \mathrm{~kW}$, and only 1 was over $10,000 \mathrm{~kW}$. Approximately $26 \mathrm{~K}$ data points were removed with this filter, resulting in a final dataset that contained $332 \mathrm{~K}$ data points across 127 sites for model training and testing activities (Figure 2). The age of the sites within the final dataset ranged from newly installed sites up to 10 years, with a majority being less than 5 years in age (Figure A2).

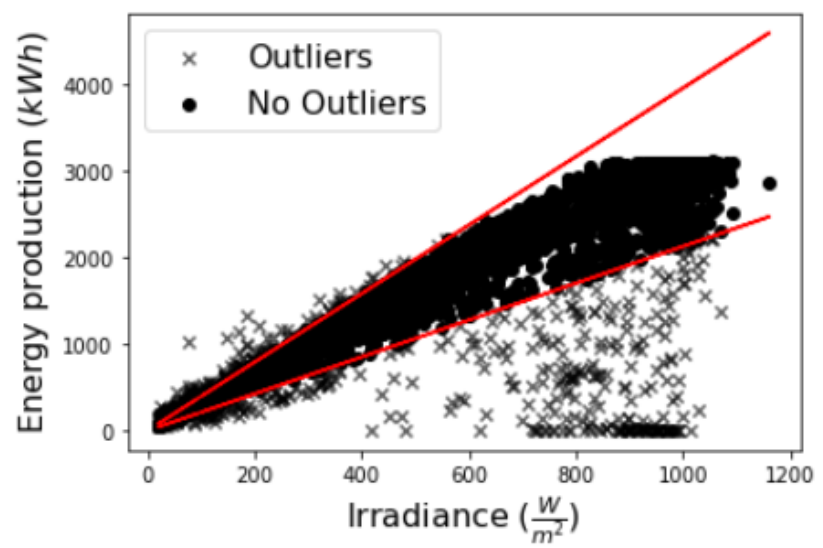

Figure 3. An example of anomaly-based filter (energy production vs. irradiance) for a particular site. Anomalous data points (visualized as Xs) are often lower than non-anomalous values within the distribution-derived bands (red lines).

\subsection{Variable Standardization}

The specific inputs used for model training mimic commonly available parameters used in current expected energy models (e.g., [15]), such as irradiance and site capacity. However, with covariates at different scales (e.g., $\left\{0 \frac{\mathrm{W}}{\mathrm{m}^{2}}<I<1.2 \times 10^{3} \frac{\mathrm{W}}{\mathrm{m}^{2}}\right\}$ while $\left\{1 \times 10^{2} \mathrm{~kW}<C_{D C}<1.3 \times 10^{5} \mathrm{~kW}\right\}$ ), variable standardization is required to reduce model sensitivity to parameter scales. In particular, without standardization, weights generated 
for each parameter are more likely to reflect scalar nuances rather than the relative importance of the parameter to the outcome of interest. Variable standardization centers the data by subtracting data points in a feature from its associated mean value $(\mu)$ and then scales the data by dividing by the associated standard deviation $(\sigma)$-i.e., $Z=\frac{\mu-\bar{\mu}}{\sigma_{X}}$. The resulting standardized variables have a mean of zero and a standard deviation of one. This process makes parameters easier to rank in terms of influence; the variable with the larger parameter holds a more important effect on the output response. Thus, variable standardization also aids in the interpretability of the derived parameters, especially when variable interactions (e.g., $I \times C_{D C}$ ) are introduced. The mean and standard deviation parameters used to standardize irradiance, capacity, and energy values are captured in Table 1.

Table 1. Mean and standard deviation (StDev) parameters used to standardize variables prior to training regression models.

\begin{tabular}{ccccc}
\hline & \multicolumn{2}{c}{$>\mathbf{1 0 0 0} \mathbf{~ k W}$ Systems } & \multicolumn{2}{c}{$<\mathbf{1 0 0 0} \mathbf{~ k W}$ Systems } \\
\cline { 2 - 5 } Parameter & Mean & StDev & Mean & StDev \\
\hline Irradiance & 571.459 & 324.199 & 413.533 & 286.110 \\
Capacity & $14,916.234$ & $20,030.000$ & 375.919 & 234.151 \\
Energy & 7449.152 & $12,054.525$ & 119.008 & 119.829 \\
\hline
\end{tabular}

\subsection{Model Design and Training}

Similar to other machine learning models, regression techniques leverage input data to learn relationships and use those relationships to predict unseen quantities. These relationships are generally contained in model parameters $(\hat{\beta})$, which map predictors, as summarized in a design matrix $\mathbf{X}$, to an output $\hat{Y}=\mathbf{X} \hat{\beta}+\epsilon$ with residual model error $\epsilon$. Many different regression techniques exist; these techniques typically vary in the structure of the cost function, which quantifies the error between predicted and expected values. This cost function $(C)$ is usually captured as a summation of loss functions (calculated on each data point) across the training set. The set $\hat{\beta}$, which renders the smallest cost, is defined as the learned parameters, mathematically notated as:

$$
\hat{\beta}=\underset{\theta}{\arg \min } C .
$$

A popular regression model is the ordinary least squares (OLS), which defines its best model $\left(\hat{\beta}_{O L S}=\underset{\theta}{\arg \min } S S E\right)$ with an objective function equal to the sum of squared errors (SSE):

$$
S S E=\sum_{i=1}^{n}\left(y_{i}-\hat{y}_{i}\right)^{2}=\sum_{i=1}^{n}\left(y-\sum_{j=0}^{p} \hat{\beta}_{j} x_{i j}\right)^{2}
$$

where $n$ is the number of samples, $p$ is the number of predictors, and $x_{i j}$ is the $i^{t h}$ value for the $j^{\text {th }}$ explanatory variable. As shown in the equation, the SSE sums the squared difference between each sample $(y)$ and its associated model estimate $(\hat{y})$. High emphasis is naturally placed on reducing high-error samples. Therefore, outliers can have a large effect on the learned parameters, so data preprocessing steps are required for robust model development. Additionally, OLS renders non-zero coefficients on all $\hat{\beta}$, which can create small, insubstantial parameters which are likely components of the training dataset and therefore contribute to model overfitting and thus should be removed from the model.

Alternate approaches to OLS include the Theil-Sen regressor [29], which is robust against outliers since it chooses the median of the slopes of all lines between pairs of points, as well as techniques such as Lasso regression [30] that explicitly address model overfitting by reducing model complexity (i.e., the number of parameters used). For this analysis, the latter was selected since Lasso regression models are able to incorporate both parameter regularization and residual sum of squares into the loss function. The cost function for 
Lasso regression $\hat{\beta}_{\text {lasso }}=\underset{\theta}{\arg \min }\left(S S E+\alpha \sum_{j=1}^{p}\left|\beta_{j}\right|\right)$ incorporates an L1 regularization term $\alpha \sum_{j=1}^{p}\left|\beta_{j}\right|$, which penalizes the magnitude of the $\beta$ terms. This penalization tends to shrink coefficients to zero, rendering a more parsimonious model; we use an $\alpha=0.003$ for defining the impact of the regularization on the regression kernel. Specifically, the penalization acts as a bias, which in turn can reduce overall error due to the bias-variance tradeoff [31].

Standardized variables are passed into Lasso regression to learn a linear model, which relates the input variables to energy. Multiple combinations of input variables were used to train the regression models (more details below). For all models, a randomized $(80-20 \%)$ split is utilized to partition the preprocessed, standardized data into train and test partitions, respectively.

In addition to individual parameter influences, interactions and temporal factors were incorporated as input features to capture nuances within the datasets. Interaction parameters, which allow the effect of one parameter on the response variable to be weighted by the value of another variable, are introduced by including terms which are the product of two or more predictor variables. For example, Figure 4 shows that the relationship between $E$ and $I$ does vary across $C_{D C}$. Thus, the inclusion of an $I$ and $C_{D C}$ interaction term may be helpful in predicting the generated energy. The suite of interaction combinations are instantiated using polynomial models up to the third order (i.e., degree $d=3$ ). In a model with $d=2$ and 2 covariates, the initiated regression model would take the following form:

$$
y=\beta_{0}+\beta_{1} x_{1}^{2}+\beta_{2} x_{2}^{2}+\beta_{3} x_{1} x_{2}+\beta_{4} x_{1}+\beta_{5} x_{2} .
$$

Notice that a $d=2$ also includes $d=1$ parameters (i.e., $\beta_{4} x_{1}$ and $\beta_{5} x_{2}$ ). This remains true for all values of the polynomial power (e.g., for a model initiated with $d=3$, terms from $d=2$ and $d=1$ are also included). Two interaction polynomial orders are tested: a second-order $(d=2)$ and a third-order $(d=3)$ (Table 2). The particular interaction noted above $\left(I \times C_{D C}\right)$ is captured in multiple models, including an additive model with a single interaction term (Table 2).

In addition to interactions, temporal factors are used to capture a variable's changing effect on the energy generated over time. For instance, the correlation between $I$ and $E$ changes over the course of the year due to spectral irradiance effects [32,33]. Therefore, allowing the model to capture time-variant nuances may be important for capturing such nonlinearities. Three temporal based conditions were explored: seasonal (four per year), monthly, and hourly. A model with two predictor variables and monthly temporal-based variable conditions would be instantiated as:

$$
\begin{aligned}
y & =a_{j a n} 1_{t \in j a n} x_{1}+a_{f e b} 1_{t \in f e b} x_{1}+\ldots+a_{d e c} 1_{t \in d e c} x_{1} \\
& +b_{j a n} 1_{t \in j a n} x_{2}+b_{f e b} 1_{t \in f e b} x_{2}+\ldots+b_{d e c} 1_{t \in d e c} x_{2}
\end{aligned}
$$

where the $a$ and $b$ parameters are coefficients describing the effect of parameter $x_{1}$ and $x_{2}$, respectively, when conditioned on a month of the year. For instance, $a_{j a n}$ describes the effect of $x_{1}$ on the $y$ response variable during the month of January. The indicator function $1_{t}$ masks the predictor variable to ensure it is within its timeframe. With the various combinations of interactions and temporal conditions, a total of 13 regression kernels were evaluated (Table 2; see Appendix A for some of the mathematical formulations). 


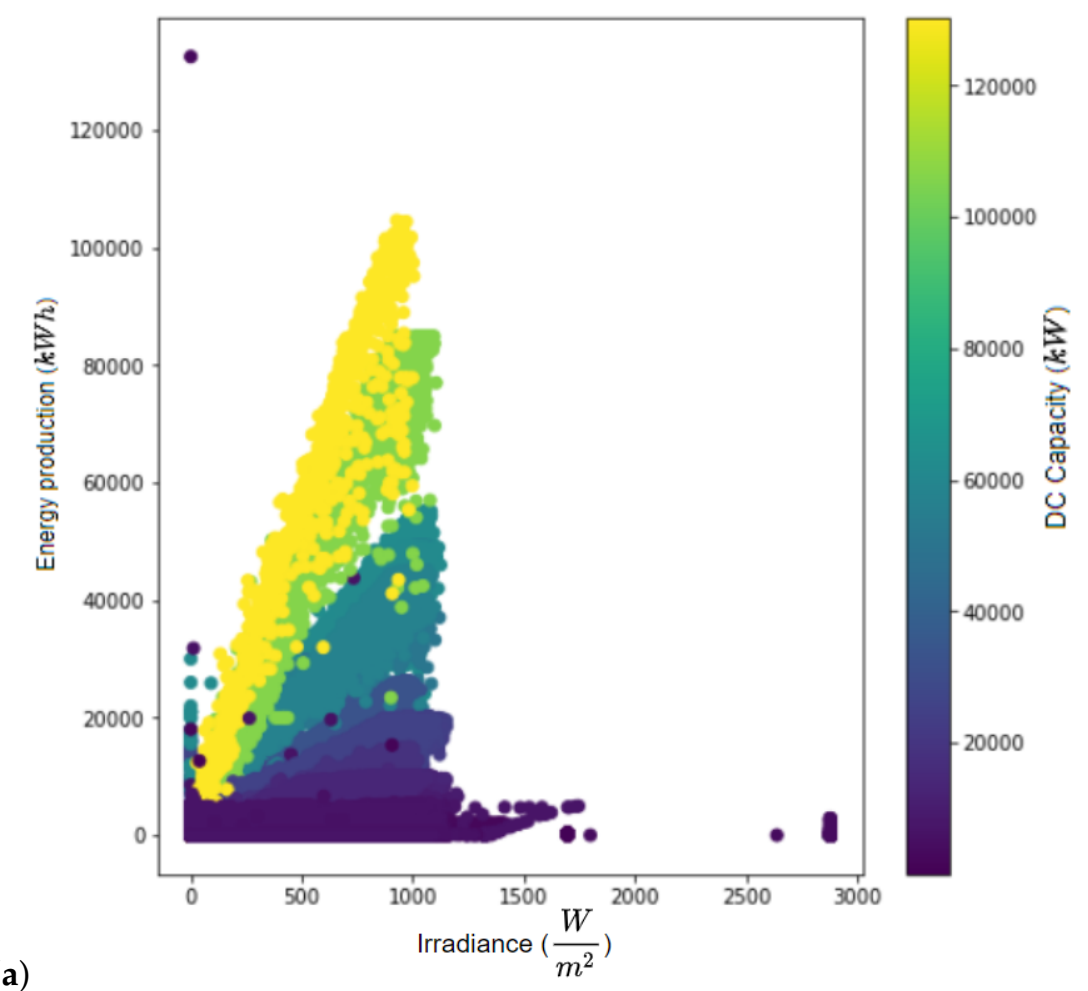

(a)

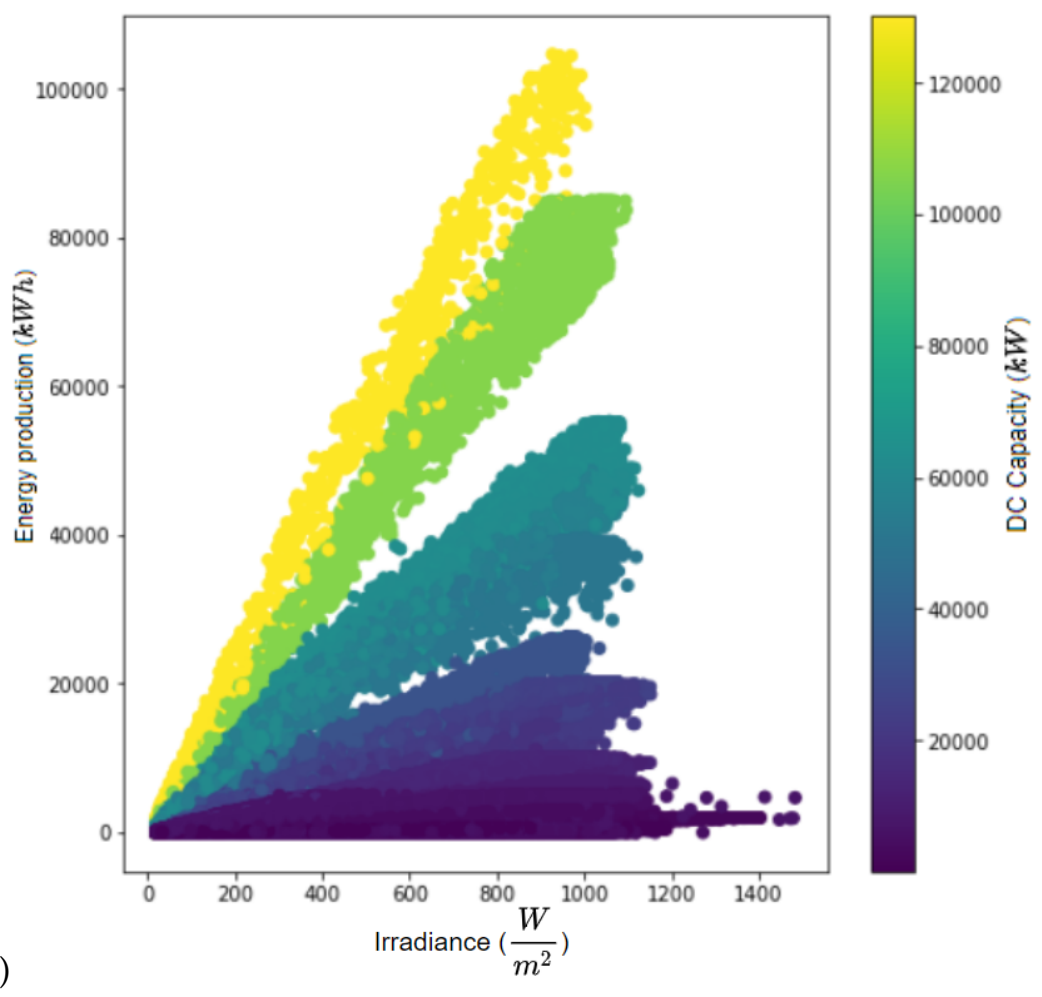

Figure 4. Correlation between energy production and irradiance for raw data (a) and preprocessed data (b) for different site capacities. Higher correlations in the preprocessed data indicate interaction between DC capacity and irradiance for energy production. 
Table 2. Combinations of parameters used to initiate the 13 regression kernels evaluated in this study. The simple additive model attaches a parameter to each predictor variable to evaluate individual effects. ${ }^{*}$ For hourly temporal conditions, only $15 \mathrm{~h}$ are used to reflect daytime hours.

\begin{tabular}{|c|c|c|c|c|c|c|}
\hline Model Category & Time Variables (t) & Interaction Degree (d) & Number Parameters for M Inputs & \multicolumn{3}{|c|}{ Number Parameters for 2 Inputs } \\
\hline Additive & & & $M+1$ & 3 & & \\
\hline Additive with interaction & & 2nd-order $(\mathrm{d}=2)$ & $M+2$ & 4 & & \\
\hline Additive with Time-weighted & $\begin{array}{l}\text { Season }(t=4) \\
\text { Month }(t=12) \\
\text { Hour }\left(t=15^{*}\right)\end{array}$ & & $t M+1$ & $\begin{array}{l}9 \\
25 \\
31\end{array}$ & & \\
\hline Polynomial & & $\begin{array}{l}\text { 2nd-order }(\mathrm{d}=2) \\
\text { 3rd-order }(\mathrm{d}=3)\end{array}$ & $\sum_{i=1}^{d} C^{R}(M, i)+1$ & $\begin{array}{l}6 \\
10 \\
\end{array}$ & & \\
\hline Polynomial with Time-weighted & $\begin{array}{l}\text { Season }(t=4) \\
\text { Month }(t=12) \\
\text { Hour }\left(t=15^{*}\right)\end{array}$ & $\begin{array}{l}\text { 2nd-order }(d=2) \\
\text { 3rd-order }(d=3)\end{array}$ & $t \sum_{i=1}^{d} C^{R}(M, i)+1$ & $\begin{array}{l}t=4 \\
t=12 \\
t=15\end{array}$ & $\begin{array}{l}\mathrm{d}=2 \\
25 \\
73 \\
90\end{array}$ & $\begin{array}{l}\mathrm{d}=3 \\
41 \\
121 \\
151\end{array}$ \\
\hline
\end{tabular}

\subsection{Model Evaluation}

Three metrics were used to evaluate the performance of the trained expected energy models: logarithmic root mean squared error (log RMSE), coefficient of determination $\left(R^{2}\right)$, and percent error $(\delta)$. Both partner-provided expected energy values and those calculated by the leading standardized expected energy model (i.e., IEC 61724) were used as reference values for model evaluations.

The root mean squared error (RMSE) is a common goodness-of-fit statistic used for model evaluation. The RMSE is expressed as:

$$
R M S E=\sqrt{\frac{1}{n} \sum_{i=1}^{n}\left(y_{i}-\hat{y}_{i}\right)^{2}}
$$

where $y_{i}$ and $\hat{y}_{i}$ are the measured and predicted values of the response variable, and $n$ is the number of samples. RMSE is in the same units as the response variable (i.e., $\mathrm{kWh}$ ). Lower RMSE values indicate a better, lower predicted error. Because the error can be quite large in magnitude $\left(10^{0}\right.$ to $\left.10^{10}\right)$, a logarithmic transform is applied to facilitate evaluations. Because the magnitude of the error is closely connected to a site's capacity, the log RMSE cannot be used to compare model performance between sites unless the sites are similar in size.

The coefficient of determination $\left(R^{2}\right)$, however, can be used to compare model performance across different site sizes. Specifically, $R^{2}$ is calculated as:

$$
R^{2}=1-\frac{\sum\left(y_{i}-\hat{y}_{i}\right)^{2}}{\sum\left(y_{i}-\bar{y}\right)^{2}}
$$

where $\bar{y}$ is the average of the $y$ values. $R^{2}$ denotes the proportion of variability in the response explained by the model with a value of 1 , indicating a perfect fit. $R^{2}$ was used to compare trained model outputs with partner-generated expected energy values, whose underlying model structures were unknown.

Generally, however, $R^{2}$ is not well-suited for comparing models across varying numbers of parameters. Thus, when comparing the 13 trained models to one another, we utilize an adjusted $R_{a d j}^{2}$ metric, which checks whether the added parameters contribute to the explanation of the predictor variable and penalizes models with unnecessary complexity [34]. Low-effect parameters (i.e., $\beta \approx 0$ ) reduce the model's overall fit score. The adjusted $R_{a d j}^{2}$ is calculated as follows:

$$
R_{a d j}^{2}=1-\left(\frac{\left(1-R^{2}\right)(n-1)}{n-p-1}\right),
$$

where $n$ is the number of samples, and $p$ is the number of predictors. 
Finally, $\delta$ was used to capture the directionality of error (i.e., overprediction vs. underprediction):

$$
\delta=100 \times \frac{\hat{y}-y}{y} .
$$

The $\log R M S E$ and $R^{2}$ were implemented to evaluate model performance at both site and fleet (i.e., across multiple sites) levels, while $\delta$ was only implemented at the fleet level; all metrics were reported on the test dataset. T-tests were used to evaluate significance in performance variations between the trained and reference values.

\section{Results and Discussion}

Data processing activities generally increased the correlations between the predictor variables (i.e., irradiance and capacity) and the response variable (i.e., energy) (Table A1). The processed data were inputted into a total of 13 trained models-ranging in model complexity (pre-lasso) from 3 parameters for the 'simple additive' model pre-lasso to 151 parameters for the 'third-order-hour' (see Table 3). Generally, the number of parameters were lower for all models post-lasso fit, except for the 'simple additive' and 'additive interaction' models, likely indicating the already sparse construction of these models.

Table 3. This table describes the parameterization and performance of all of the models evaluated in the results of this paper. Wins are summarized by showing the percentage of sites where a given model was the top performer according to the associated goodness-of-fit metric (Adj. $R^{2}$ or $\log R M S E)$; the IEC model was used as the reference value for $\log R M S E$ calculations. The thirdorder interactions model and basic model perform consistently well, a conclusion also found on the heatmaps (Figure 5). Additionally, because lasso regression was leveraged, the models decrease in size after training the model.

\begin{tabular}{|c|c|c|c|c|c|c|c|c|}
\hline \multirow[b]{2}{*}{ Models } & \multirow[b]{2}{*}{ Time Variable } & \multirow[b]{2}{*}{$\begin{array}{c}\text { Interaction } \\
\text { Degree }\end{array}$} & \multirow[b]{2}{*}{$\begin{array}{c}\text { Number } \\
\text { Parameters } \\
\text { Pre-Lasso }\end{array}$} & \multirow[b]{2}{*}{$\begin{array}{l}\text { Number } \\
\text { Parameters } \\
\text { Post-Lasso }\end{array}$} & \multicolumn{2}{|c|}{$>1000$ kW Systems } & \multicolumn{2}{|c|}{$<1000$ kW Systems } \\
\hline & & & & & Wins: Adj. $\mathbf{R}^{2}$ & $\begin{array}{l}\text { Wins: } \\
\text { logRMSE }\end{array}$ & Wins: Adj. $\mathbf{R}^{2}$ & $\begin{array}{c}\text { Wins: } \\
\text { logRMSE }\end{array}$ \\
\hline $\begin{array}{l}\text { Third-order } \\
\text { interactions }\end{array}$ & & 3 & 10 & 9 & 47.9 & 49.0 & 38.7 & 38.7 \\
\hline $\begin{array}{l}\text { Additive } \\
\text { interaction }\end{array}$ & & 1 & 4 & 4 & 22.9 & 17.7 & 32.3 & 29.0 \\
\hline $\begin{array}{l}\text { Second-order } \\
\text { interactions }\end{array}$ & & 2 & 6 & 5 & 15.6 & 10.4 & 6.5 & 0.0 \\
\hline $\begin{array}{l}\text { Second-order } \\
\text { seasonal }\end{array}$ & season & 2 & 25 & 18 & 9.4 & 15.6 & 3.2 & 3.2 \\
\hline $\begin{array}{c}\text { Third-order } \\
\text { seasonal }\end{array}$ & season & 3 & 41 & 27 & 1.0 & 4.2 & 3.2 & 3.2 \\
\hline $\begin{array}{l}\text { Second-order } \\
\text { month }\end{array}$ & month & 2 & 73 & 43 & 0.0 & 0.0 & 3.2 & 3.2 \\
\hline $\begin{array}{l}\text { Second-order } \\
\text { hour }\end{array}$ & hour & 2 & 90 & 44 & 0.0 & 0.0 & 0.0 & 0.0 \\
\hline $\begin{array}{c}\text { Third-order } \\
\text { month }\end{array}$ & month & 3 & 121 & 68 & 0.0 & 0.0 & 0.0 & 3.2 \\
\hline $\begin{array}{c}\text { Third-order } \\
\text { hour }\end{array}$ & hour & 3 & 151 & 81 & 1.0 & 1.0 & 0.0 & 6.5 \\
\hline IEC & & & & & 1.0 & 1.0 & 12.9 & 12.9 \\
\hline hour & hour & 1 & 31 & 26 & 0.0 & 0.0 & 0.0 & 0.0 \\
\hline month & month & 1 & 25 & 25 & 0.0 & 0.0 & 0.0 & 0.0 \\
\hline seasonal & season & 1 & 9 & 9 & 1.0 & 1.0 & 0.0 & 0.0 \\
\hline $\begin{array}{l}\text { simple } \\
\text { additive }\end{array}$ & & 1 & 3 & 3 & 0.0 & 0.0 & 0.0 & 0.0 \\
\hline
\end{tabular}


Initially, the various models were trained using data across all system sizes. However, this approach demonstrated systemic underperformance for low-capacity systems $(<1000 \mathrm{~kW}$ DC capacity). Specifically, the best trained models (i.e., 'third-order interactions' and 'additive interaction') outperformed the IEC model in terms of log RMSE when tested on every single system above $1300 \mathrm{~kW}$ DC capacity; however, 12 of 34 systems below a $1300 \mathrm{~kW}$ DC capacity underperformed relative to the IEC model. This result likely reflects the varying relationships between the site DC capacity and the energy generated; systems of higher capacity tend to receive a higher maximum energy generated per DC capacity (Figure A1). To better deal with this varying linearity, two separate sets of models were trained: one for models under $1000 \mathrm{~kW}$ DC capacity and another over.

Across both high-capacity and low-capacity systems, models with the $I \times C_{D C}$ interaction term perform better than those without the interaction term (i.e., 'hour', 'month', 'seasonal', and 'simple additive' models). For example, two of the top-performing models (across both high-capacity and low-capacity systems) are the 'simple additive' and the 'third-order interactions' models, both of which contain this interaction term (Table 3 and Figure 5). The 'additive interaction' trained (AIT) model has four parameters:

$$
\hat{e}= \begin{cases}0.07+0.69 i+0.65 c+0.42 i c & C_{D C}<1000 \mathrm{~kW} \\ -0.06+0.29 i+0.76 c+0.40 i c & C_{D C} \geq 1000 \mathrm{~kW}\end{cases}
$$

where $i$ and $c$ define the standardized irradiance and capacity variables, respectively, as defined in Table 1. The 'third-order interactions' model, on the other hand, contains these four terms as well as higher-order interactions (e.g., irradiance ${ }^{2} \times$ capacity, capacity $^{3}$ ). Although the variables within both of these two models are similar to the IEC standard, the inclusion of the interaction term, which highlights that the linear relationship between $I$ and $E$ is moderated by $C_{D C}$ (Figure 4), likely explains the superior performance of these models relative to that standard. The heatmaps of the $\log R M S E$ values highlight the evaluation metric's dependence on site capacity (Figure 5a,c), while the adjusted $R^{2}$ heatmaps show consistent performance across site capacity (Figure $5 b$,d). The vertical concentration of dark bars likely reflects data quality issues not addressed by data preprocessing steps. A comparison of the associated partner generated expected energy estimates to those predicted from models also demonstrates that the AIT-derived estimates have lower average percent errors than the other models and the IEC (Table A2). Further evaluation of 2 years of records at a single site demonstrates that the AIT-derived estimates have a lower standard deviation and do not overestimate as much as the IEC-derived estimates (Figure A3). Given its parsimonious nature, the AIT model is subjected to further evaluation for both highand low-capacity systems. 
(a): Log RMSE for high-capacity systems:

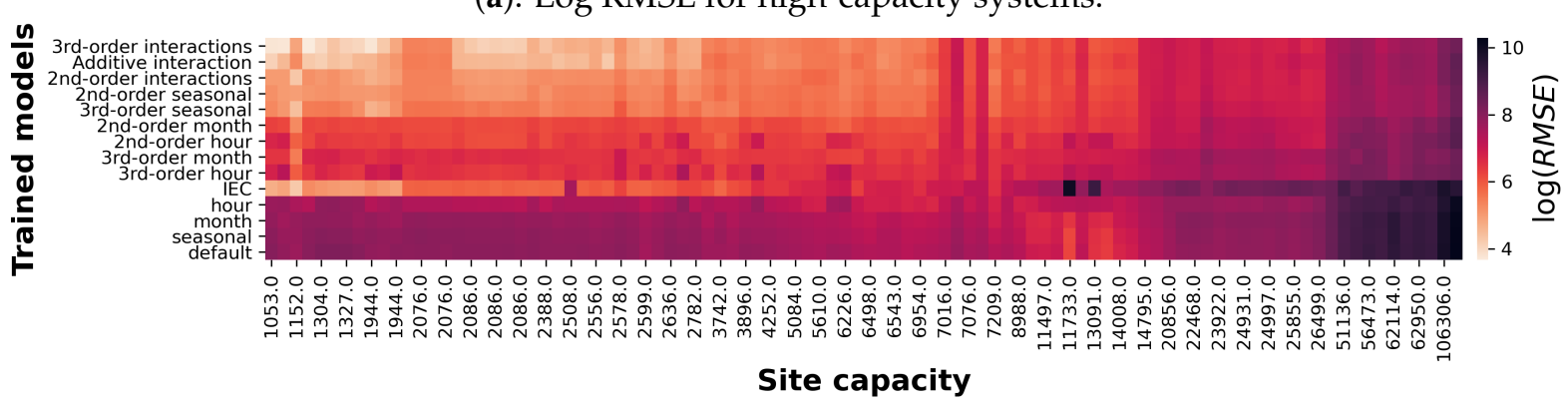

(b): Adjusted $R^{2}$ for high-capacity systems:

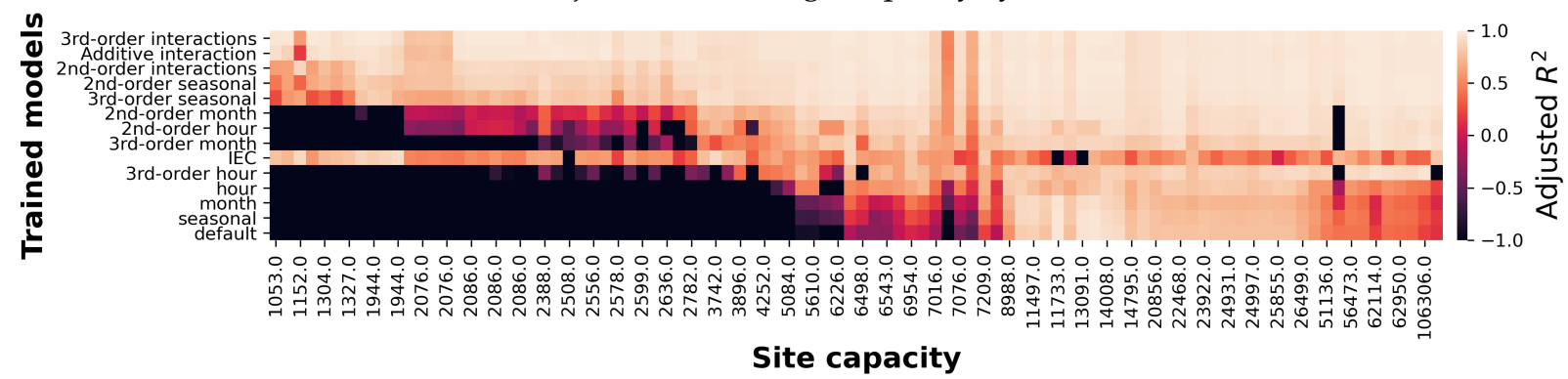

(c): Log RMSE for low-capacity systems:

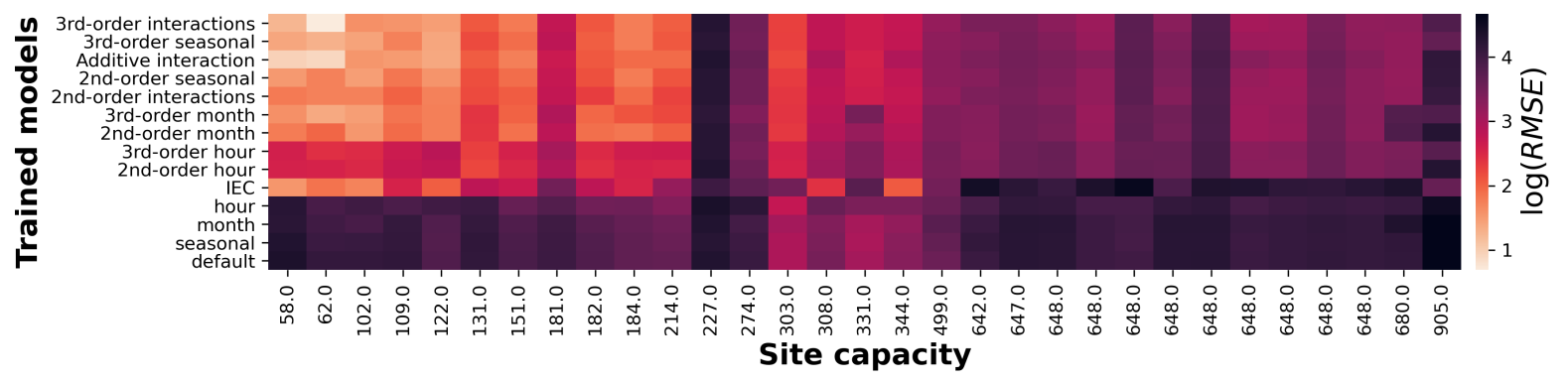

(d): Adjusted $R^{2}$ for low-capacity systems:

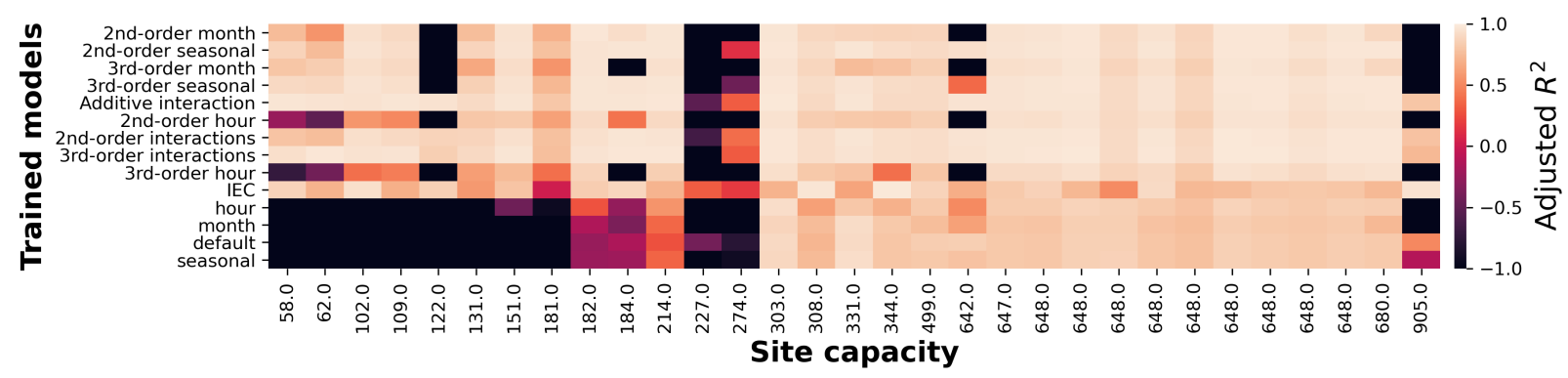

Figure 5. High-capacity (a,b) and low-capacity (c,d) site-level model evaluations with test data. Models with lighter colors (i.e., low values for $\log R M S E$ and values closer to 1 for adjusted $R^{2}$ ) indicate better performance.

\subsection{High-Capacity Systems}

For high-capacity systems, a significant (almost uniform) difference is found in both the $\log R M S E$ and $R^{2}$ values between the AIT and the IEC reference models (Figure 6a,b). Across the sites, the AIT model improves the goodness of fit by 0.42 in $R^{2}$ (IEC: 0.501; AIT: 0.93 ) and 1.16 in $\log$ RMSE (IEC: 6.99; AIT: 5.83). Generally, there are very few systems for which the IEC model performs better than the trained models (Table 3). The percent error $(\delta)$, on average, of the AIT model (3.65) is significantly lower than the IEC model (20.86) for high-capacity systems. An evaluation of percent error shows that the AIT generally 
performs well (i.e., $\delta \approx 0$ and thinner standard deviation bars) for most irradiance levels, except at the two extremes (i.e., $<200$ and $>1100 \frac{\mathrm{W}}{\mathrm{m}^{2}}$ ) (Figure 7).

The difference in performance relative to the IEC model is especially pronounced for larger system sizes (Figure 6a,b). Although the AIT model appears to show a small improvement over the partner-provided values (Figure $6 \mathrm{a}, \mathrm{b}$ ), a $t$-test concluded that the distributions of both the $\log R M S E$ ( $p$-value: 0.78 ) and $R^{2}$ ( $p$-value: 0.48 ) are not significantly different.

(a): High-capacity systems:

High-capacity sites (> 1000kW)
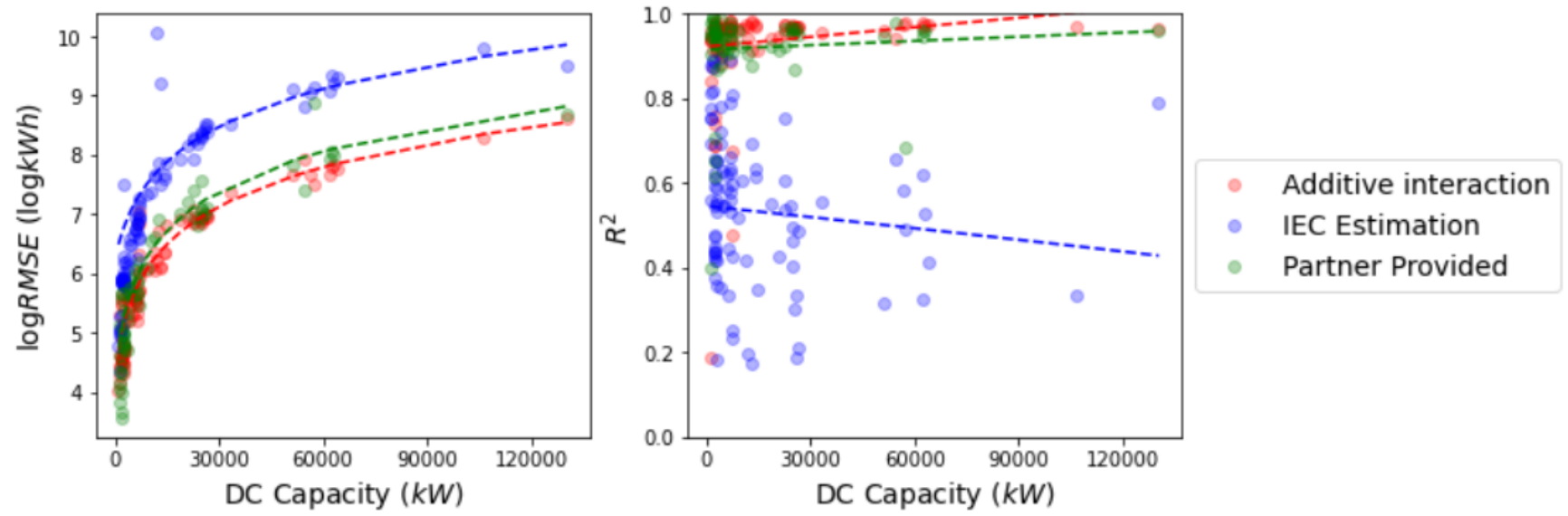

(b): Low-capacity systems:

Low-capacity sites $(<1000 \mathrm{~kW})$
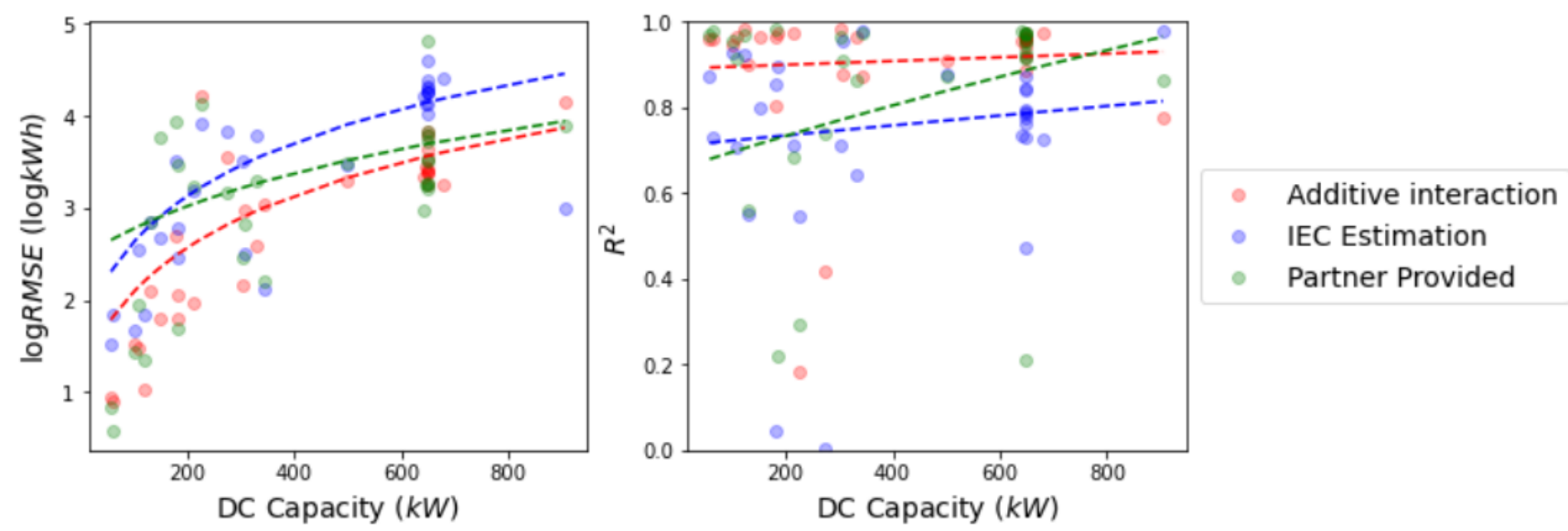

Figure 6. Model evaluation using $\log R M S E$ and $R^{2}$ metrics for high-capacity systems (a) and lowcapacity systems (b). Data points reflect site-level summaries of associated test data while dotted lines reflect best line fits to support visual pattern identification. The $R^{2}$ metric was used for this analysis (vs. adjusted $R^{2}$ ) since partner-provided model architectures are unknown. The 'additive interaction' regression model (in red) is comparable to the partner-provided proprietary values (green) and consistently performs better than the IEC standard (blue), especially at higher capacity values. 
(a) High-capacity systems:

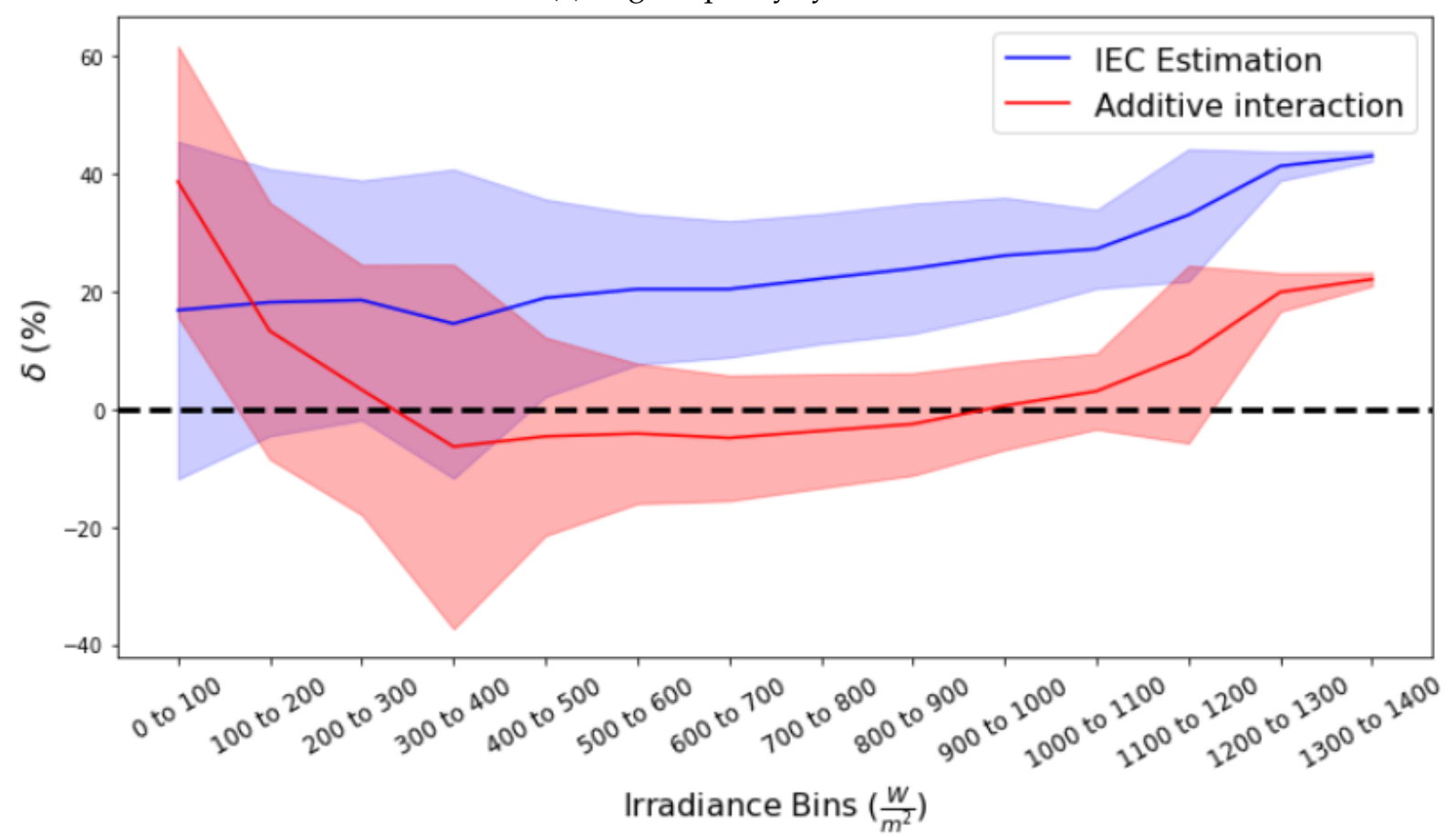

(b): Low-capacity systems:

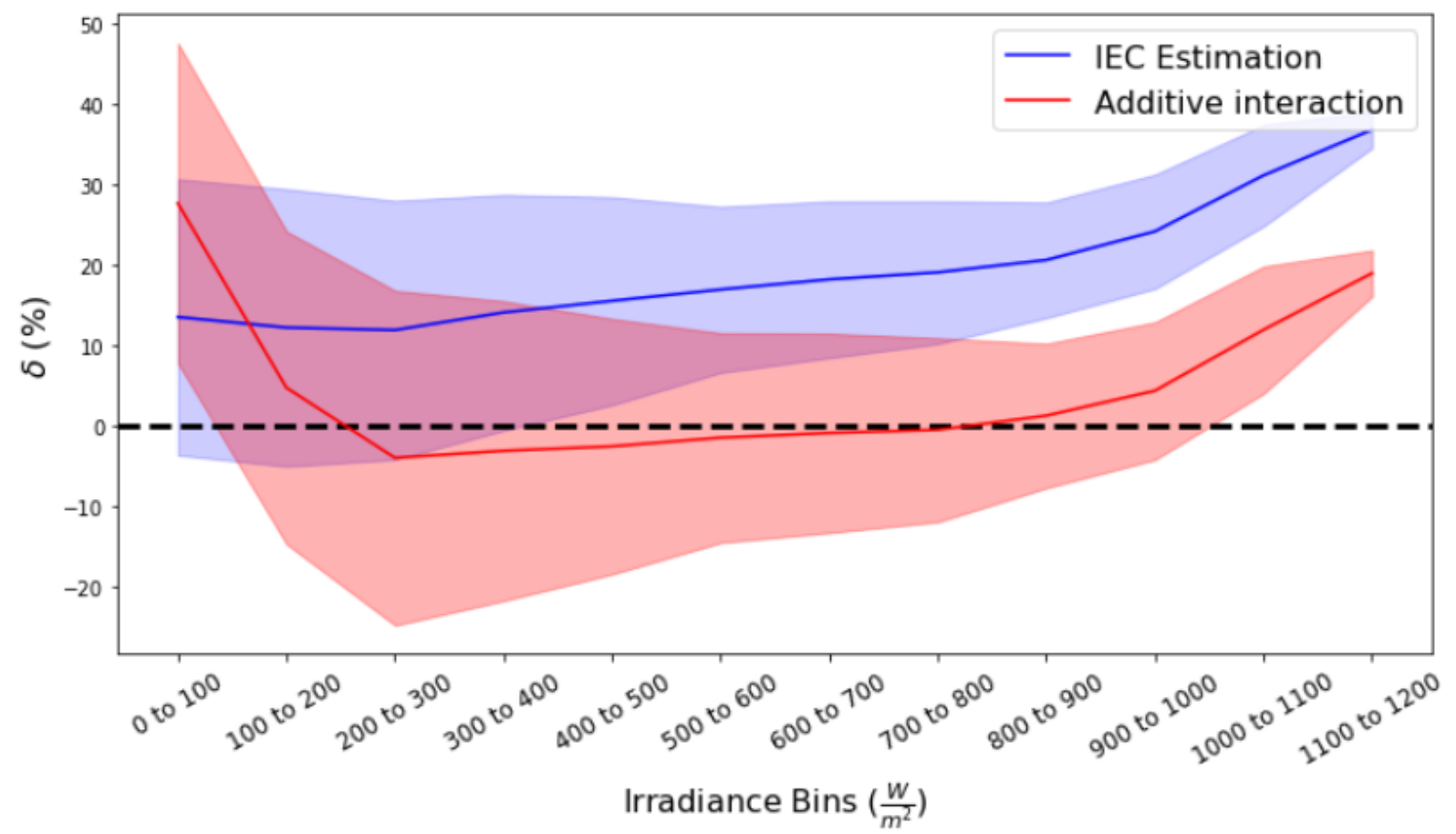

Figure 7. Percent error as a function of irradiance shows that the 'additive interaction' model outperforms the IEC standard across both high-capacity (a) and low-capacity (b) systems. Lines indicate mean values, while shaded region captures one standard deviation. The 'additive interaction' model performs best $(\delta \approx 0)$ at $500-1100 \frac{\mathrm{W}}{\mathrm{m}^{2}}$ and at 200-1000 $\frac{\mathrm{W}}{\mathrm{m}^{2}}$ for high-capacity and low-capacity systems, respectively.

\subsection{Low-Capacity Systems}

The model performance of the AIT for low-capacity systems was generally comparable to that of high-capacity systems, although the improvements were not as high. Across all low-capacity sites, the AIT model's goodness of fit improved by 0.165 in $R^{2}$ (IEC: 0.74; AIT: 
0.90 ) and 0.61 in $\log$ RMSE (IEC: 3.35; AIT: 2.75) (Figure 6a,b). Out of the 31 low-capacity sites (comprising 50K hours), the IEC-based model outperformed the trained models in 4 systems (Table 3 ). In some of the low-capacity systems, the measured energy is much higher than expected (Figure 5). The tendency of the IEC model to overpredict likely describes why this model performs better for some of the lower-capacity systems.

The $\delta$, on average, is 4.42 and 15.37 for the AIT model and IEC model, respectively. Similar to the high-capacity system, the percent error values are greater at the extremes (Figure $7 \mathrm{~b}$ ). However, the standard error is generally higher in the low-capacity systems, as evidenced by wider standard deviation bars across the irradiance levels (Figure $7 \mathrm{~b}$ ).

\subsection{Limitations and Future Work}

The methodological approach of this analysis was strongly guided by available data. However, future work could extend these methods to consider: (1) energy generation at finer resolutions, (2) additional co-variates, and (3) alternate model formulations. For example, scaling could be used to consider alternate frequencies (beyond the hourly intervals considered in this study) post-evaluation. The methods used in this analysis explicitly omitted variables not included in current standard models (e.g., [15]). However, future assessments could more explicitly incorporate co-variates such as temperature, wind speed, and even age of the site. The latter would especially enable active consideration of degradation influence, which can influence long-term energy generation of PV sites [35]. Additional co-variates (such as type of inverters and modules) could also be included in subsequent iterations to capture more subtle impacts associated with differing site designs. Finally, future work could consider alternate model formulations (e.g., splines) to improve model continuity and better capture energy generation for smaller system sizes.

\section{Conclusions}

This work demonstrates the opportunities for leveraging data-driven, machine learning methods to generate more robust expected energy models. Generally, when compared to partner-provided values, the trained regression models outperform the IEC standard, especially in high-capacity systems. Detailed evaluation of the parsimonious AIT or 'additive interaction' model, in particular, demonstrated significant potential for use as a standardized, fleet-level expected energy model. The specific code used to train the regression models as well as the AIT model have been integrated with pvOps, an open source Python package which supports the evaluation of field data by PV researchers and operators; pvOps can be accessed at https://github.com/sandialabs/pvOps, accessed on 22 December 2021. Although this work presents findings specific to PV systems, the general methodologies can be applied to any domain that uses expected energy models to support site planning and O\&M activities. Ongoing evaluations and improvements of these standardized expected energy models will continue to increase the accuracy and precision of site-level PV performance evaluations, which is critical to supporting reliability and economic assessments of PV operations and maintenance.

Supplementary Materials: The following supporting information can be downloaded at https: / / www.mdpi.com/article/10.3390/app12041872/s1. The supplementary material also includes a subsection with mathematical models for top-performing trained models.

Author Contributions: Conceptualization, T.G.; methodology, M.W.H. and T.G.; software, M.W.H.; validation, M.W.H. and T.G.; data curation, M.W.H.; writing - original draft preparation, M.W.H.; writing-review and editing, T.G.; visualization, M.W.H.; supervision, T.G.; project administration, T.G.; funding acquisition, T.G. All authors have read and agreed to the published version of the manuscript.

Funding: This research was funded by U.S. Department of Energy Solar Energy Technologies Office (Award No. 34172).

Institutional Review Board Statement: Not applicable. 
Informed Consent Statement: Not applicable.

Data Availability Statement: The raw data was procured under non-disclosure agreements and thus, cannot be shared. However, an anonymized version of the filtered dataset used in this study analysis can be found within the article and Supplementary Information.

Acknowledgments: The authors would like to thank our industry partners for sharing data in support of this analysis as well as Sam Gilletly for their assistance with reviewing an earlier version of this manuscript. Sandia National Laboratories is a multimission laboratory managed and operated by National Technology and Engineering Solutions of Sandia LLC, a wholly owned subsidiary of Honeywell International Inc. for the U.S. Department of Energy's National Nuclear Security Administration under contract DE-NA0003525. The views expressed in the article do not necessarily represent the views of the U.S. Department of Energy or the United States Government.

Conflicts of Interest: The authors declare no conflict of interest.

\section{Abbreviations}

The following abbreviations are used in this manuscript:

E Energy $(\mathrm{kWh})$

$\hat{E} \quad$ Expected energy $(\mathrm{kWh})$

$I \quad$ irradiance $\left(\frac{\mathrm{W}}{\mathrm{m}^{2}}\right)$

$C_{D C} \quad$ DC capacity $(\mathrm{kW})$

$T_{a m b}$ ambient temperature $\left({ }^{\circ} \mathrm{C}\right)$

$T_{\text {mod }} \quad$ module temperature $\left({ }^{\circ} \mathrm{C}\right)$

e $\quad$ standardized $E$ (see Table 1)

$i \quad$ standardized $I$ (see Table 1 )

c standardized C (see Table 1)

RMSE Root mean squared error

$n \quad$ number of samples

$p \quad$ number of predictors

\section{Appendix A}

Appendix A.1. Tables E Figures

Table A1. Correlations with energy generation and regression model parameters pre- and post- data processing. Correlations with energy production are generally comparable with irradiance across raw and filtered data, while correlations for site capacity are significantly higher for the filtered data than the raw data.

\begin{tabular}{cccc}
\hline & \multicolumn{3}{c}{ Data Subsets } \\
\cline { 2 - 4 } Parameters & Raw & $\begin{array}{c}\text { Post-Data Quality } \\
\text { Filters }\end{array}$ & $\begin{array}{c}\text { Post-System } \\
\text { Anomaly Filters }\end{array}$ \\
\hline Irradiance $(I)$ & 0.46 & 0.41 & 0.40 \\
\hline Capacity $\left(C_{D C}\right)$ & 0.55 & 0.85 & 0.89 \\
\hline
\end{tabular}

Table A2. Summary of average percent errors for each of the trained models, using the partnergenerated values as the reference value.

\begin{tabular}{ccc}
\hline & \multicolumn{3}{c}{$\begin{array}{c}\text { Average Percent Error } \\
\mathbf{1 0 0 0} \mathbf{~ k W ~ S y s t e m s ~}\end{array}$} \\
\hline Third-order interactions & 0.03 & 0.23 \\
\hline Second-order interactions & 0.03 & 0.21 \\
\hline Third-order seasonal & 0.05 & 0.20 \\
\hline Second-order seasonal & 0.04 & 0.16 \\
\hline
\end{tabular}


Table A2. Cont.

\begin{tabular}{|c|c|c|}
\hline & \multicolumn{2}{|c|}{ Average Percent Error } \\
\hline & $>1000$ kW Systems & $\leq 1000 \mathrm{~kW}$ Systems \\
\hline Third-order month & 0.12 & 0.27 \\
\hline Second-order month & 0.08 & 0.27 \\
\hline Third-order hour & 0.13 & 0.15 \\
\hline Second-order hour & 0.10 & 0.05 \\
\hline Additive interaction & 0.03 & 0.00 \\
\hline simple additive & 0.36 & 1.44 \\
\hline IEC & 0.39 & 1.75 \\
\hline hour & 0.28 & 1.01 \\
\hline month & 0.32 & 1.30 \\
\hline seasonal & 0.32 & 1.55 \\
\hline
\end{tabular}

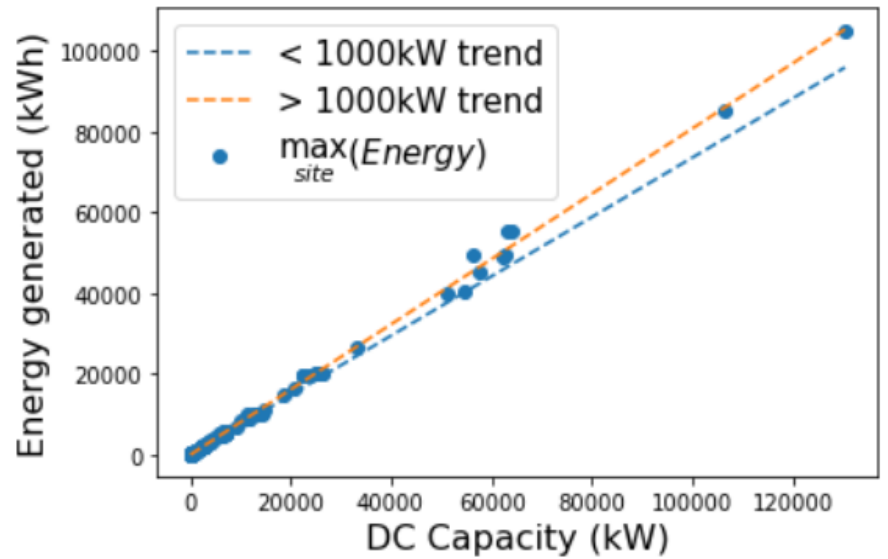

Figure A1. Relationship between DC capacity $(\mathrm{kW})$ and hourly generated energy $(\mathrm{kWh})$. The blue dots show a site's DC capacity versus its maximum recorded energy generated in a single hour. Although the trends are largely linear, the slopes differ for sites smaller than $1000 \mathrm{~kW}$ (blue dashed lines) and sites larger than $1000 \mathrm{~kW}$ (orange dashed lines). Since slight deviations in slope can render large prediction error, we train two separate model based on site size.

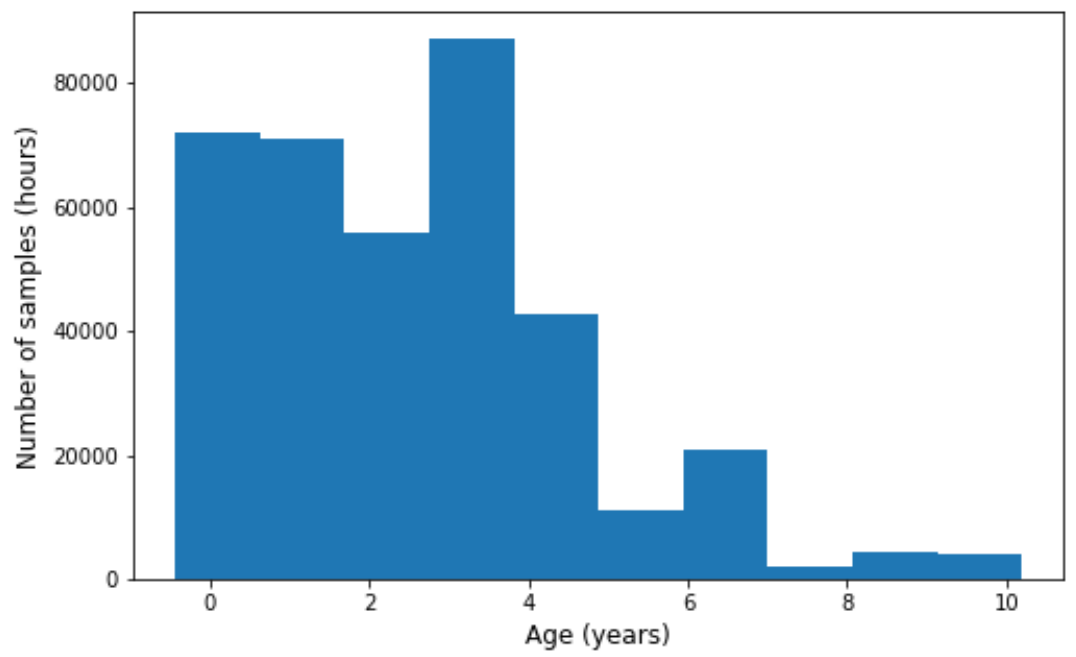

Figure A2. Age of sites within the final dataset. A majority are less than five years of age. 


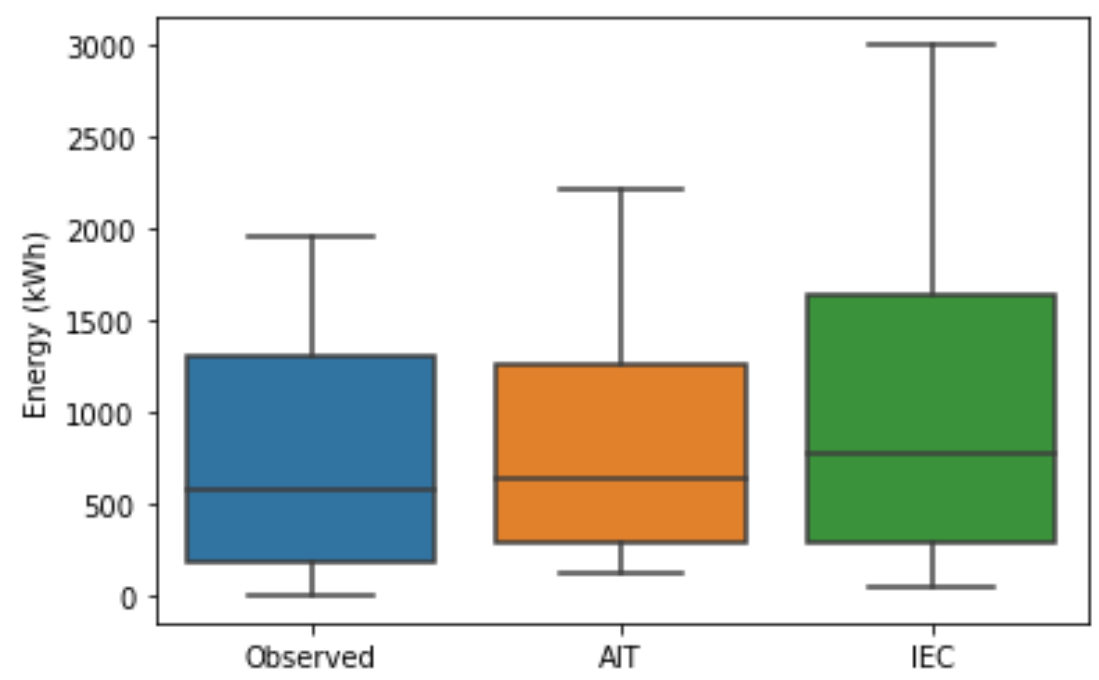

Figure A3. A 2-year comparison of observed energy with expected energy estimates from the trained additive interaction trained (AIT) model and the IEC standard at a single site. The IEC-derived estimates overestimate much more so than AIT-derived estimates. Slightly higher values of AITderived estimates relative to observed values likely indicate local failures at the site.

\section{Appendix A.2. Top-Performing Trained Models}

Mathematical equations associated with the trained regression models. In general, these model formulations contain more parameters and do not perform as well as the additive interaction model. High-capacity refers to systems with greater than or equal to $1000 \mathrm{~kW}$ in $C_{D C}$ while low-capacity refers to systems smaller than $1000 \mathrm{~kW}$ in $C_{D C}$.

High-capacity second-order seasonal:

$$
\begin{aligned}
e & =0.309 i I_{\text {winter }}+0.292 i I_{\text {spring }}+0.281 i I_{\text {summer }}+0.287 i I_{\text {fall }} \\
& +0.762 c I_{\text {winter }}+0.734 c I_{\text {spring }}+0.724 c I_{\text {summer }}+0.730 c I_{\text {fall }} \\
& -0.003 i^{2} I_{\text {spring }}-0.009 i^{2} I_{\text {summer }}-0.007 i^{2} I_{\text {fall }}+0.430 i c I_{\text {winter }} \\
& +0.397 i c I_{\text {spring }}+0.380 i c I_{\text {summer }}+0.384 i c I_{\text {fall }}+0.008 c^{2} I_{\text {winter }} \\
& +0.008 c^{2} I_{\text {spring }}-0.003 c^{2} I_{\text {fall }}-0.054
\end{aligned}
$$

High-capacity third-order interactions:

$$
e=0.293 i+0.769 c-0.019 i^{2}+0.394 i c+0.021 i^{2} c+0.004 i c^{2}+0.001 c^{3}-0.039
$$

High-capacity third-order seasonal:

$$
\begin{aligned}
e & =0.265 i I_{\text {winter }}+0.270 i I_{\text {spring }}+0.252 i I_{\text {summer }}+0.243 i I_{\text {fall }} \\
& +0.749 c I_{\text {winter }}+0.740 c I_{\text {spring }}+0.722 c I_{\text {summer }}+0.730 c I_{\text {fall }} \\
& -0.006 i^{2} I_{\text {summer }}-0.388 i c I_{\text {winter }}+0.378 i c I_{\text {spring }}+0.365 i c I_{\text {summer }} \\
& +0.346 i c I_{\text {fall }}+0.019 c^{2} I_{\text {winter }}+0.005 c^{2} I_{\text {spring }}-0.002 c^{2} I_{\text {fall }} \\
& +0.016 i^{3} I_{\text {winter }}+0.008 i^{3} I_{\text {spring }}+0.014 i^{3} I_{\text {summer }}+0.017 i^{3} I_{\text {fall }} \\
& -0.013 i c^{2} I_{\text {winter }}+0.008 i c^{2} I_{\text {spring }}+0.007 i c^{2} I_{\text {summer }}+0.019 i c^{2} I_{\text {fall }} \\
& -0.002 c^{3} I_{\text {winter }}-0.054
\end{aligned}
$$


High-capacity second-order interactions:

$$
e=0.295 i+0.745 c-0.018 i^{2}+0.404 i c+0.004 c^{2}-0.042
$$

Low-capacity second-order seasonal:

$$
\begin{aligned}
e & =0.711 i I_{\text {winter }}+0.686 i I_{\text {spring }}+0.680 i I_{\text {summer }}+0.714 i I_{\text {fall }} \\
& +0.617 c I_{\text {winter }}+0.576 c I_{\text {spring }}+0.605 c I_{\text {summer }}+0.636 c I_{\text {fall }} \\
& -0.046 i^{2} I_{\text {winter }}-0.028 i^{2} I_{\text {spring }}-0.046 i^{2} I_{\text {summer }}-0.038 i^{2} I_{\text {fall }} \\
& +0.401 i c I_{\text {winter }}+0.381 i c I_{\text {spring }}+0.382 i c I_{\text {summer }}+0.427 i c I_{\text {fall }} \\
& +0.001 c^{2} I_{\text {winter }}-0.006 c^{2} I_{\text {summer }}-0.104
\end{aligned}
$$

Low-capacity third-order interactions:

$$
e=0.746 i+0.642 c-0.043 i^{2}+0.426 i c-0.019 i^{3}-0.034 i^{2} c-0.017 c^{3}+0.115
$$

Low-capacity third-order seasonal:

$$
\begin{aligned}
e & =0.757 i I_{\text {winter }}+0.684 i I_{\text {spring }}+0.667 i I_{\text {summer }}+0.712 i I_{\text {fall }} \\
& +0.577 c I_{\text {winter }}+0.486 c I_{\text {spring }}+0.567 c I_{\text {summer }}+0.619 c I_{\text {fall }} \\
& -0.023 i^{2} I_{\text {winter }}-0.027 i^{2} I_{\text {spring }}-0.050 i^{2} I_{\text {summer }}-0.033 i^{2} I_{\text {fall }} \\
& +0.413 i c I_{\text {winter }}+0.382 i c I_{\text {spring }}+0.381 i c I_{\text {summer }}+0.428 i c I_{\text {fall }} \\
& -0.028 i^{3} I_{\text {winter }}-0.007 i^{3} I_{\text {summer }}-0.022 i^{2} c I_{\text {winter }}-0.004 i^{2} c I_{\text {fall }} \\
& -0.047 c^{3} I_{\text {winter }}+0.068 c^{3} I_{\text {spring }}+0.027 c^{3} I_{\text {summer }}+0.016 c^{3} I_{\text {fall }}+0.099
\end{aligned}
$$

Low-capacity second-order interactions:

$$
e=0.719 i+0.630 c-0.063 i^{2}+0.411 i c-0.124
$$

\section{References}

1. Golnas, A. PV system reliability: An operator's perspective. In Proceedings of the 2012 IEEE 38th Photovoltaic Specialists Conference (PVSC) PART 2, Austin, TX, USA, 3-8 June 2012; pp. 1-6.

2. Feng, M.; Bashir, N.; Shenoy, P.; Irwin, D.; Kosanovic, D. SunDown: Model-driven Per-Panel Solar Anomaly Detection for Residential Arrays. In Proceedings of the 3rd ACM SIGCAS Conference on Computing and Sustainable Societies, Guayaquil, Ecuador, 15-17 June 2020; pp. 291-295.

3. Dyamond, W.; Rix, A. Detecting Anomalous Events for a Grid Connected PV Power Plant Using Sensor Data. In Proceedings of the 2019 Southern African Universities Power Engineering Conference/Robotics and Mechatronics/Pattern Recognition Association of South Africa (SAUPEC/RobMech/PRASA), Bloemfontein, South Africa, 29-31 January 2019; pp. 287-292.

4. Harrou, F.; Sun, Y.; Taghezouit, B.; Saidi, A.; Hamlati, M.E. Reliable fault detection and diagnosis of photovoltaic systems based on statistical monitoring approaches. Renew. Energy 2018, 116, 22-37. [CrossRef]

5. Platon, R.; Martel, J.; Woodruff, N.; Chau, T.Y. Online fault detection in PV systems. IEEE Trans. Sustain. Energy 2015, 6, $1200-1207$. [CrossRef]

6. $\quad$ Blair, N.; Dobos, A.P.; Freeman, J.; Neises, T.; Wagner, M.; Ferguson, T.; Gilman, P.; Janzou, S. System Advisor Model, Sam 2014.1. 14: General Description; Technical Report; National Renewable Energy Lab.: Golden, CO, USA, 2014.

7. Deline, C.; Anderson, K.; Jordan, D.; Walker, A.; Desai, J.; Perry, K.; Muller, M.; Marion, B.; White, R. PV Fleet Performance Data Initiative: Performance Index-Based Analysis; Technical Report; National Renewable Energy Lab.: Golden, CO, USA, 2021.

8. Gilletly, S.; Jackson, N.; Staid, A. Quantifying Wildfire-Induced Impacts to Photovoltaic Energy Production in the western United States. In Proceedings of the 2021 IEEE 48th Photovoltaic Specialists Conference (PVSC), Online, 20-25 June 2021.

9. Klise, G.T.; Stein, J.S. Models Used to Assess the Performance of Photovoltaic Systems; Sandia National Laboratories: Albuquerque, NM, USA, 2009.

10. De Soto, W.; Klein, S.A.; Beckman, W.A. Improvement and validation of a model for photovoltaic array performance. Sol. Energy 2006, 80, 78-88. [CrossRef] 
11. Arab, A.H.; Taghezouit, B.; Abdeladim, K.; Semaoui, S.; Razagui, A.; Gherbi, A.; Boulahchiche, S.; Mahammed, I.H. Maximum power output performance modeling of solar photovoltaic modules. Energy Rep. 2020, 6, 680-686. [CrossRef]

12. King, D.L.; Kratochvil, J.A.; Boyson, W.E. Photovoltaic Array Performance Model; Sandia National Laboratories: Albuquerque, NM, USA, 2004.

13. Boyson, W.E.; Galbraith, G.M.; King, D.L.; Gonzalez, S. Performance Model for Grid-Connected Photovoltaic Inverters; Technical Report; Sandia National Laboratories: Albuquerque, NM, USA, 2007.

14. Dobos, A.P. PVWatts Version 5 Manual; Technical Report; National Renewable Energy Lab.: Golden, CO, USA, 2014.

15. ISO/IEC TR 29110-1:2016; Photovoltaic System Performance-Part 1: Monitoring. International Electrotechnical Commission: Geneva, CH, USA, 2017.

16. Myers, D. Evaluation of the Performance of the Pvusa Rating Methodology Applied to Dual Junction pv Technology: Preprint (Revised); Technical Report; National Renewable Energy Lab.: Golden, CO, USA, 2009.

17. DNV GL. Narrowing the Performance Gap: Reconciling Predicted and Actual Energy Production; Technical Report; DNV GL: Oakland, CA, USA, 2019.

18. Hanawalt, S. The Challenge of Perfect Operating Data; Technical Report; Power Factors: San Francisco, CA, USA, 2020.

19. Livera, A.; Theristis, M.; Makrides, G.; Sutterlueti, J.; Ransome, S.; Georghiou, G.E. Performance Analysis of Mechanistic and Machine Learning models for Photovoltaic energy yield prediction. In Proceedings of the 36th European Photovoltaic Solar Energy Conference and Exhibition, Marseille, France, 9-13 September 2019; pp. 9-13.

20. Ascencio-Vásquez, J.; Bevc, J.; Reba, K.; Brecl, K.; Jankovec, M.; Topič, M. Advanced PV Performance Modelling Based on Different Levels of Irradiance Data Accuracy. Energies 2020, 13, 2166. [CrossRef]

21. Wang, S.; Zhang, Y.; Zhang, C.; Yang, M. Improved artificial neural network method for predicting photovoltaic output performance. Glob. Energy Interconnect. 2020, 3, 553-561. [CrossRef]

22. Bertsimas, D.; Delarue, A.; Jaillet, P.; Martin, S. The price of interpretability. arXiv 2019, arXiv:1907.03419.

23. Klise, G.T. PV Reliability Operations Maintenance (PVROM) Database: Data Collection \& Analysis Insights; Technical Report; Sandia National Lab.: Albuquerque, NM, USA, 2015.

24. Klise, K.A.; Stein, J.S.; Cunningham, J. Application of IEC 61724 Standards to Analyze PV System Performance in Different Climates. In Proceedings of the 2017 IEEE 44th Photovoltaic Specialist Conference (PVSC), Washington, DC, USA, 25-30 June 2017; pp. 3161-3166.

25. Klise, K.A. Performance Monitoring Using Pecos; Technical Report; Sandia National Lab.: Albuquerque, NM, USA, 2016.

26. IEC TS 61724-3:2016; Photovoltaic System Performance-Part 3: Energy Evaluation Method. International Electrotechnical Commission: Geneva, CH, USA, 2016.

27. Vining, W.; Hansen, C. PVAnalytics. 2021. Available online: https://github.com/pvlib/pvanalytics (accessed on 22 December 2021).

28. Malik, S.; Dassler, D.; Fröbel, J.; Schneider, J.; Ebert, M. Outdoor data evaluation of half-/full-cell modules with regard to measurement uncertainties and the application of statistical methods. In Proceedings of the 29th European Photovoltaic Solar Energy Conference and Exhibition, Amsterdam, The Netherlands, 22-26 September 2014; pp. 3269-3273.

29. Theil, H. A rank-invariant method of linear and polynomial regression analysis. Indag. Math. 1950, $12,173$.

30. Tibshirani, R. Regression shrinkage and selection via the lasso: A retrospective. J. R. Stat. Soc. Ser. B 2011, 73, 273-282. [CrossRef]

31. Geman, S.; Bienenstock, E.; Doursat, R. Neural networks and the bias/variance dilemma. Neural Comput. 1992, 4, 1-58. [CrossRef]

32. Dirnberger, D.; Blackburn, G.; Müller, B.; Reise, C. On the impact of solar spectral irradiance on the yield of different PV technologies. Sol. Energy Mater. Sol. Cells 2015, 132, 431-442. [CrossRef]

33. Eke, R.; Betts, T.R. Spectral irradiance effects on the outdoor performance of photovoltaic modules. Renew. Sustain. Energy Rev. 2017, 69, 429-434. [CrossRef]

34. Arias, A.L. R-Squared, Adjusted R-Squared, the F Test, and Multicollinearity. In Understanding Regression Analysis; Chapman and Hall/CRC: Boca Raton, FL, USA, 2020; pp. 185-200.

35. Jordan, D.C.; Deline, C.; Kurtz, S.R.; Kimball, G.M.; Anderson, M. Robust PV degradation methodology and application. IEEE J. Photovoltaics 2017, 8, 525-531. [CrossRef] 